\title{
Is the T1/2N1 ( $\leq 3$ nodes) category actually stage IIIA (TNM)/ IIIa (Japanese classification) in patients with primary colorectal cancer?
}

\author{
MASAYA MUKAI $^{1}$, KYOKO KISHIMA ${ }^{1}$, HIROSHI FUKUMITSU ${ }^{1}$, YASUTOMO SEKIDO ${ }^{2}$, \\ HIDEKI IZUMI $^{1}$, TATSUHIKO HOSHIKAWA ${ }^{1}$, TAKAYUKI TAJIMA ${ }^{1}$, KOUSUKE TOBITA ${ }^{1}$, \\ SOTARO SADAHIRO $^{3}$, SEIEI YASUDA ${ }^{3}$ and KYOJI OGOSHI ${ }^{3}$ \\ Departments of ${ }^{1}$ Surgery and ${ }^{2}$ Pathology, Tokai University Hachioji Hospital, Hachioji, Tokyo 192-0032; \\ ${ }^{3}$ Department of Surgery, Tokai University School of Medicine, Isehara, Kanagawa 259-1193, Japan
}

Received February 28, 2011; Accepted April 4, 2011

DOI: $10.3892 /$ or.2011.1280

\begin{abstract}
The 5-year relapse-free survival rate (5Y-RFS) and the 5-year overall survival rate (5Y-OS) were calculated for 972 patients (stage I, 206 patients; stage II, 396 patients; stage III, 370 patients). We divided the stage III group into 259 patients with IIIa/N1 disease ( $\leq 3$ positive nodes) and 111 patients with IIIb/N2 disease ( $\geq 4$ positive nodes) according to the Japanese classification. The IIIa/N1 and IIIb/N2 categories were each subdivided into T1/2 (stage IIIa, 45 cases; IIIb, 9 cases) and $\geq \mathrm{T} 3$ (stage IIIa, 214 cases; IIIb, 102 cases) according to the TNM classification, and 5Y-RFS and 5Y-OS were compared between each subcategory and each group. The $5 \mathrm{Y}-\mathrm{RFS} / 5 \mathrm{Y}-\mathrm{OS}$ values calculated for each stage were as follows: stage I, 94.0/90.7\%; stage II, 80.5/81.1\%; stage III, 63.5/65.7\%. When stage IIIa was compared with IIIb, we obtained $67.9 / 72.0 \%$ for stage IIIa and $53.6 \%(\mathrm{p}=0.001) / 50.4 \%$ $(\mathrm{p}<0.001)$ for stage IIIb. For stage IIIa vs. IIIb in the $\geq \mathrm{T} 3$ category, we obtained $63.1 / 68.5 \%$ for stage IIIa and $51.9 \%$ $(\mathrm{p}=0.010) / 49.0 \%(\mathrm{p}=0.008)$ for stage IIIb. For stage IIIa vs. IIIb in the T1/2 category, we obtained $92.1 / 92.0 \%$ for stage IIIa and $72.9 \%(\mathrm{p}=0.040) / 63.5 \%(\mathrm{p}=0.003)$ for stage IIIb. There were significant differences between $\mathrm{T} 1 / 2$ and $\geq \mathrm{T} 3$ within stage IIIa $(p=0.001 / p=0.009)$, but not within stage IIIb. These results suggest that the T1/2N1 category of colorectal cancer
\end{abstract}

Correspondence to: Dr Masaya Mukai, Department of Surgery, Tokai University Hachioji Hospital, Ishikawa-cho 1838, Hachioji, Tokyo 192-0032, Japan

E-mail: mukai.masaya@hachioji-hosp.tokai.ac.jp

Abbreviations: 5Y-RFS, 5-year relapse-free survival; 5Y-OS, 5-year overall survival; GRCPSC, General Rules for Clinical and Pathological Studies on Cancer of the Colon, Rectum and Anus; 5-FU, 5-fluorouracil; LV, leucovorin; CPT-11, irinotecan; UFT, tegafur/uracil; Uzel, oral calcium folinate; PSK, krestin

Key words: colorectal cancer, TNM classification, stage IIIA, $\mathrm{T} 1 / 2 \mathrm{~N} 1$ category should be classified as a subcategory of stage IB/Ib rather than stage IIIA (TNM)/IIIa (Japanese classification).

\section{Introduction}

In recent years, the outcome of patients with primary colorectal cancer in Japan has shown marked improvement with the development of more effective surgical techniques and adjuvant modalities such as chemotherapy and radiation therapy. It has been reported that the 5-year survival rate of patients after curative resection of Dukes' B/stage II colorectal cancer without lymph node metastases is approximately $80-85 \%$ (colon, $84.5 \pm 2.8 \%$; rectum, $79.8 \pm 4.0 \%$ ) (1-3). On the other hand, the 5-year survival rate of patients with Duke's C/ stage III cancer and lymph node metastases is approximately

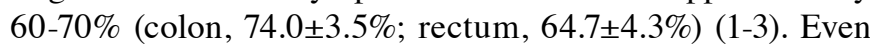
after curative resection, approximately $30-40 \%$ of these patients eventually suffer the life-threatening development of distant metastasis/recurrence. According to the TNM classification, colorectal cancer patients with lymph node metastasis are classified as stage III, which is further divided into stage IIIA (T1/2N1, $\leq 3$ metastatic nodes), stage IIIB (T3/4aN1) and stage IIIC based on the number of metastatic nodes (4). In the 7 th edition of the General Rules for Clinical and Pathological Studies on Cancer of the Colon, Rectum and Anus (GRCPSC; Japanese classification) published in 2006, considerable changes have been made. Thus, instead of the Japanese classification being based on the sites of lymph node metastasis, a more specific classification based on the number of lymph node metastases is now employed. According to the revised rules, N1 patients ( $\leq 3$ nodes) and N2 patients ( $\geq 4$ nodes) are classified as stage IIIa and IIIb, respectively, regardless of their $\mathrm{T}$ factor (5). Lymph node metastasis in breast cancer patients is generally considered to be a manifestation of systemic disease, therefore breast cancer stages are subcategorized according to the number of involved lymph nodes. This also reflects the viewpoint that, in various cancers, the number of lymph node metastases is an important prognostic factor that determines the outcome and the survival of the patient $(4,6-8)$. 
In Japan, the leading cause of cancer-related death for women is colorectal cancer, followed by lung, breast and gastric cancers. For men, the leading cause of death is lung cancer, followed by gastric cancer, colorectal cancer and cancer of the hepatobiliary tract. Although colorectal cancer is currently in third place, it is expected to rank first for both men and women in the near future considering its rapid increase in incidence (9). Among all gastrointestinal cancers, gastric cancer ranked far higher than the others as the leading cause of death for both men and women until around the year 2000. However, early diagnosis and early treatment of gastric cancer have led to a significant improvement in the overall outcome, decreasing both morbidity and mortality. At the same time, the number of patients with colorectal cancer has increased dramatically due to the adoption of a Westernized diet and lifestyle. In addition, there has been an increase in other types of cancer, such as breast and prostate, both of which are associated with hormonal secretion. As a result, the causes of death in Japan currently resemble those in the US and Europe (9).

The TNM classification is widely used as it is considered to be the global gold standard for clinical staging of cancer, and the GRCPSC has recently become considerably similar to the TNM classification (5). In patients with esophageal cancer, T1/2N1 disease is classified as stage IIB according to the TNM classification and as stage II according to the Japanese classification of esophageal cancer. In the case of gastric cancer, T1N1 and T2N1 disease are subcategorized into stage IB and stage IIA, respectively, according to both the TNM classification and the Japanese classification of gastric carcinoma. Thus, it is common for stage I/II disease, which are determined by $\mathrm{T}$ and $\mathrm{N}$ factors, to be classified into subcategories $(4,5)$.

However, there have been no studies involving the classification of $\mathrm{T} 1 / 2 \mathrm{~N} 1$ colorectal cancer into stage I/II subcategories based on the TNM classification or the Japanese classifications for other types of cancer in patients who have undergone curative resection of node-positive stage III primary colorectal cancer. Therefore, the purpose of this study was to examine whether the T1/2N1 category ( $\leq 3$ nodes) of stage III colorectal cancer should be subcategorized as stage I or stage II.

\section{Patients and methods}

In the 15-year period between January 1995 and January 2010 , 1,014 patients received curative resection of primary colorectal cancer. Among them, a total of 972 patients (pathological stage I, 206 cases; stage II, 396 cases; and stage III, 370 cases) met the following criteria: i) age $\leq 75$ years and performance status of 0 or 1 ; ii) complete medical records were available and recurrence as well as survival could be determined; and iii) the number of lymph node metastases was clearly identifiable. All stage III cases were subdivided into 259 cases of stage IIIa (N1, $\leq 3$ metastatic nodes) and 111 cases of stage IIIb (N2, $\geq 4$ metastatic nodes) based on the GRCPSC regardless of their $\mathrm{T}$ factors (5). These patients were further subdivided by the depth of tumor invasion based on the TNM classification of $\mathrm{T}$ factors, so that 45 patients from the stage IIIa group were categorized as T1/T2 and the remaining 214 patients were categorized as $\geq \mathrm{T} 3$. Similarly, 9 patients in stage IIIb were categorized as T1/T2 and the remaining 102 patients were categorized as $\geq \mathrm{T} 3$ (4). The 5-year relapse-free survival
(5Y-RFS) and the 5-year overall survival (5Y-OS) were then calculated for each category.

Postoperative treatment and follow-up. In general, stage I patients received surgery and follow-up without postoperative adjuvant therapy $(2,3,10)$, while stage II patients received postoperative adjuvant chemotherapy with oral UFT/PSK for 12 months or longer (11-13). Stage III patients received intravenous 5-FU/LV or 5-FU/LV in combination with CPT-11 for 6 months after surgery, and then received oral UFT/Uzel or UFT/ PSK for 12 months or longer (14-17). In principle, surgical resection was chosen as the first-line treatment for postoperative recurrence/metastasis. Patients who were not indicated for surgery received anticancer agents other than those mentioned above as second-line treatment with radiation therapy for pelvic local recurrence $(17,18)$. The outpatient follow-up system included measurement of tumor markers three times per year and US/CT twice a year for stage I patients, with 3-4 general examinations per year for stage II/III patients. Detection of recurrence/metastasis was confirmed by US/CT $(17,18)$.

Statistical analysis. The 5Y-RFS and 5Y-OS were calculated using the Kaplan-Meier method and the log-rank test, and hazard ratios $(95 \% \mathrm{CI})$ were used for comparison between two groups. $\mathrm{P}<0.05$ was considered to indicate a significant difference in all analyses, which were performed with SPSS 17.0 J software (SPSS Japan, Inc., Tokyo, Japan).

\section{Results}

The 5Y-RFS rate calculated for each stage was as follows: stage I $(n=206), 94.0 \%$; stage II $(n=396), 80.5 \%$; stage III $(n=370), 63.5 \%$. The results of statistical analysis were as follows: stage I vs. II: p<0.001; HR, 0.284 (95\% CI, 0.144$0.559)$; stage I vs. III: p<0.001; HR, 0.154 (95\% CI, 0.080-0.298); and stage II vs. III: p<0.001; HR, $0.543(95 \%$ CI, 0.409-0.720). The 5Y-OS calculated for each stage was as follows: stage I, 90.7\%; stage II, 81.1\%; and stage III, $65.7 \%$. The results of statistical analysis were as follows: stage I vs. II: $\mathrm{p}=0.005$; HR, 0.384 (95\% CI, 0.206-0.718); stage I vs. III: $\mathrm{p}<0.001$; HR, 0.210 (95\% CI, 0.115-0.383); and stage II vs. III: $\mathrm{p}<0.001$; HR, 0.547 (95\% CI, 0.405-0.739) (Fig. 1).

When stage IIIa $(n=259)$ and IIIb $(n=111)$ were compared, the 5Y-RFS calculated for each stage was 67.9 and $53.6 \%$, respectively ( $\mathrm{p}=0.001$; HR, $0.643 ; 95 \% \mathrm{CI}, 0.466-0.886)$, while the 5 Y-OS for each stage was 72.0 and $50.4 \%$, respectively (p<0.001; HR, 0.579; 95\% CI, 0.411-0.815) (Fig. 2).

Among patients with $\geq \mathrm{T} 3$, stage IIIa $(\mathrm{n}=214)$ was compared with IIIb ( $\mathrm{n}=102)$. The 5Y-RFS was 63.1 and $51.9 \%$, respectively $(\mathrm{p}=0.010$; HR, $0.727 ; 95 \% \mathrm{CI}, 0.527-1.003)$, while the $5 \mathrm{Y}-\mathrm{OS}$ was 68.5 and $49.0 \%$, respectively $(\mathrm{p}=0.008$; HR, 0.670 ; 95\% CI, 0.472-0.950) (Fig. 3).

Among T1/2 patients, stage IIIa $(n=45)$ was also compared with IIIb $(\mathrm{n}=9)$. The 5Y-RFS was 92.1 and $72.9 \%$, respectively ( $\mathrm{p}=0.040$; HR, 0.200; 95\% CI, 0.032-1.240), while the 5Y-OS rate was 92.0 and $63.5 \%$, respectively $(\mathrm{p}=0.003$; HR, 0.133 , 95\% CI, 0.026-0.687) (Fig. 4). 


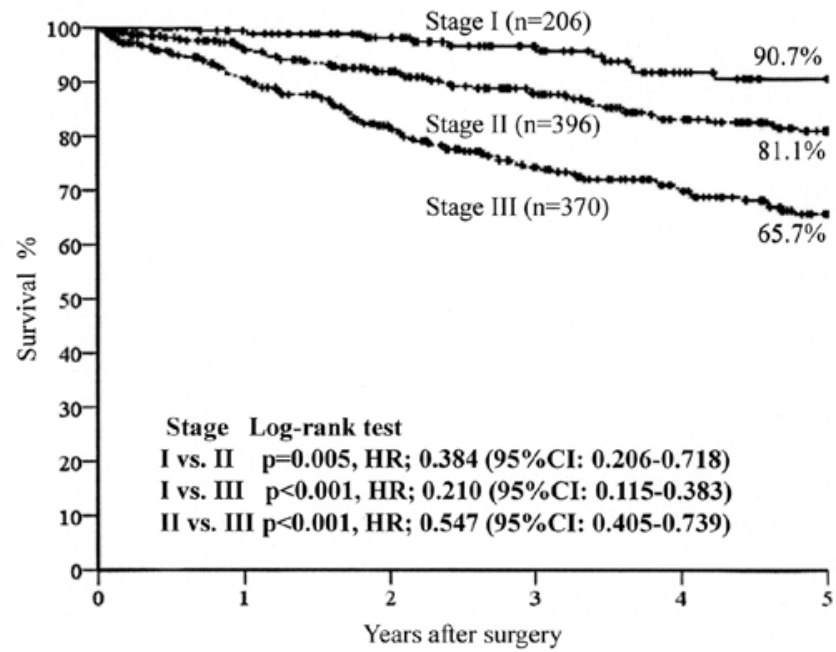

Figure 1. 5Y-OS of stage I/II/III was as follows: stage I ( $\mathrm{n}=206), 90.7 \%$ stage II ( $\mathrm{n}=396), 81.1 \%$; and stage III $(\mathrm{n}=370), 65.7 \%$. Stage I vs. II: $\mathrm{p}=0.005$ HR, 0.384 (95\% CI, 0.206-0.718); stage I vs. III: p<0.001; HR, 0.210 (95\% CI, 0.115-0.383); stage II vs. III: p<0.001; HR, 0.547 (95\% CI, 0.405-0.739).

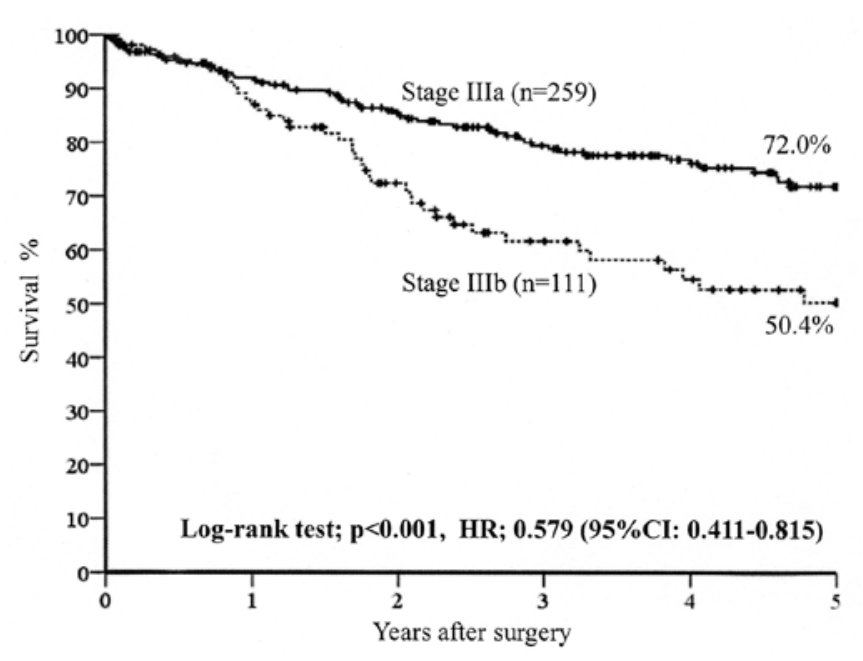

Figure 2. 5 Y-OS of stage IIIa $(\mathrm{n}=259)$ vs. IIIb $(\mathrm{n}=111)$ was $72.0 / 50.4 \%$ : $\mathrm{p}<0.001 ; \mathrm{HR}, 0.579$ (95\% CI, 0.411-0.815).

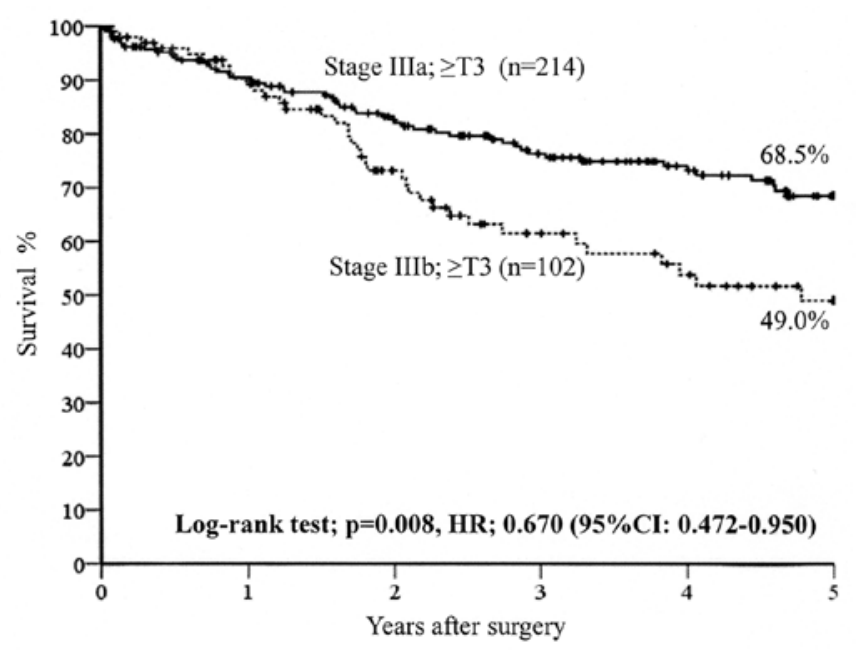

Figure 3. 5 Y-OS of $\geq \mathrm{T} 3$ patients in stage IIIa $(\mathrm{n}=214)$ vs. IIIb $(\mathrm{n}=102)$ was 68.5/49.0\%: $\mathrm{p}=0.008$; HR, 0.670 (95\% CI, 0.472-0.950).

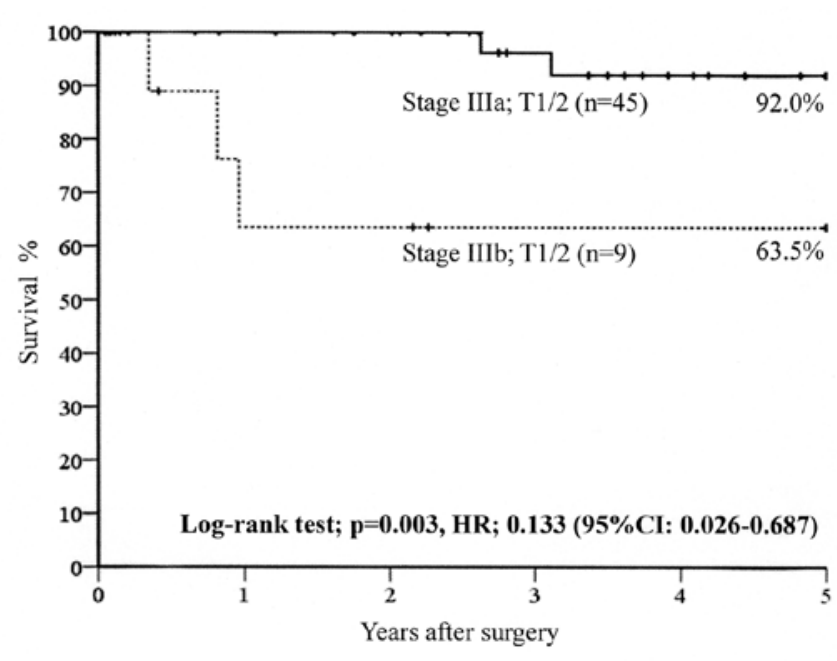

Figure 4. 5 Y-OS of T1/2 patients in stage IIIa $(n=45)$ vs. IIIb $(n=9)$ was 92.0/63.5\%: $\mathrm{p}=0.003$; HR, 0.133 (95\% CI, 0.026-0.687).

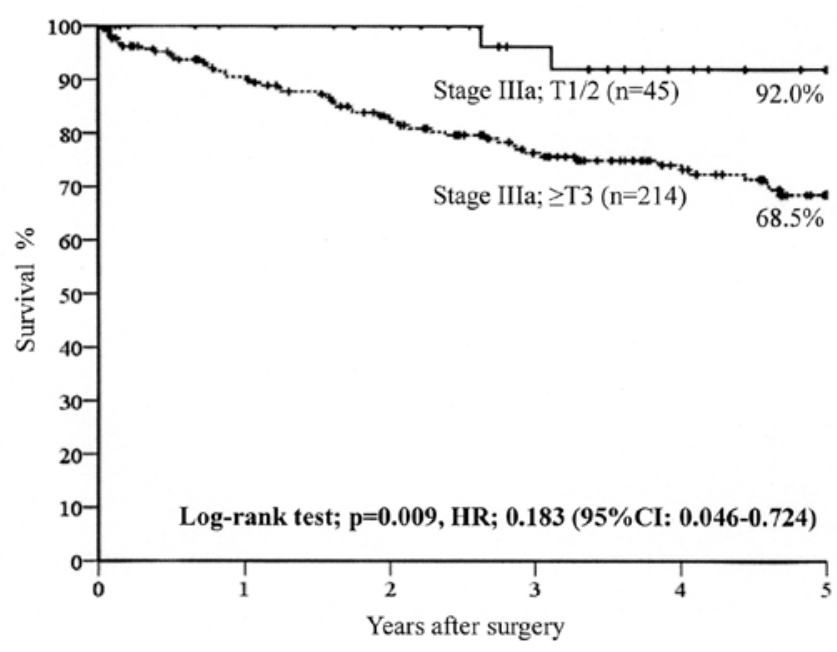

Figure 5. 5 Y-OS of stage IIIa patients with $\mathrm{T} 1 / 2(\mathrm{n}=45)$ vs. $\geq \mathrm{T} 3(\mathrm{n}=214)$ was 92.0/68.5\%: $\mathrm{p}=0.009$; HR, 0.183 (95\% CI, 0.046-0.724).

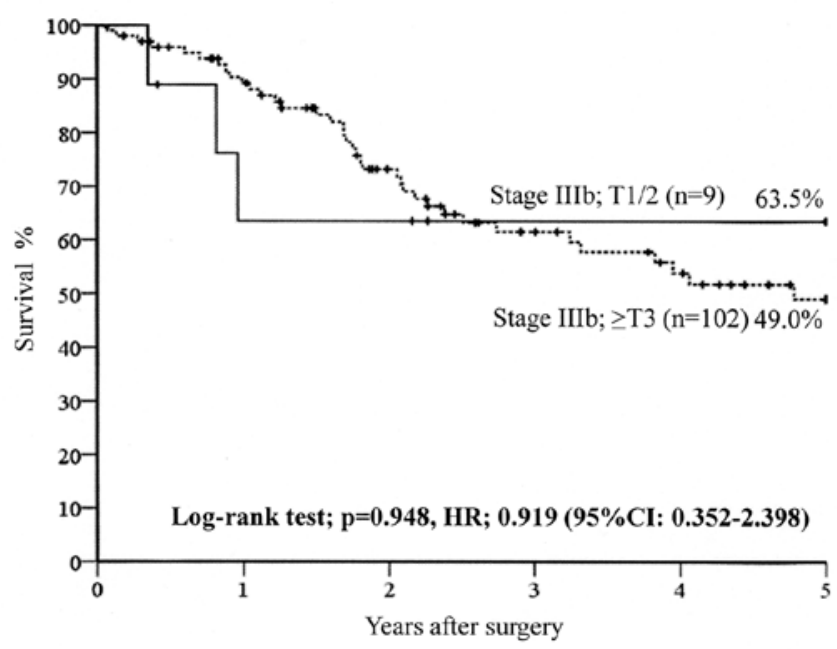

Figure 6. 5 Y-OS of stage IIIb patients with $\mathrm{T} 1 / 2(\mathrm{n}=9)$ vs. $\geq \mathrm{T} 3(\mathrm{n}=102)$ was 63.5/49.0\%: $\mathrm{p}=0.948$ (NS); HR, 0.919 (95\% CI, 0.352-2.398). 
Among stage IIIa patients, $\mathrm{T} 1 / 2(\mathrm{n}=45)$ was compared with $\geq \mathrm{T} 3$ ( $\mathrm{n}=214)$. As a result, the 5Y-RFS was 92.1 and $63.1 \%$, respectively ( $\mathrm{p}=0.001 ; \mathrm{HR}, 0.156 ; 95 \% \mathrm{CI}, 0.040-0.614)$, while $5 \mathrm{Y}-\mathrm{OS}$ was 92.0 and $68.5 \%$, respectively $(\mathrm{p}=0.009 ; \mathrm{HR}, 0.183$; 95\% CI, 0.046-0.724) (Fig. 5).

Finally, stage IIIb patients in $\mathrm{T} 1 / 2(\mathrm{n}=9)$ were compared with those in $\geq \mathrm{T} 3(\mathrm{n}=102)$. The 5Y-RFS was 72.9 and $51.9 \%$, respectively [p=0.394 (N.S.); HR, 0.567; 95\% CI, 0.163-1.970], while the 5Y-OS was 63.5 and $49.0 \%$, respectively [p $=0.948$ (N.S.); HR, 0.919; 95\% CI, 0.352-2.398] (Fig. 6).

\section{Discussion}

For cancers such as esophageal cancer, gastric cancer, breast cancer and lung cancer, stage I/II cases defined by their $\mathrm{T}$ factor (T1/2) and $\mathrm{N}$ factor (N1) are often divided into subcategories according to both the TNM and Japanese classifications. For colorectal cancer, however, all N1/2 patients are classified as stage III regardless of their T factors according to both the TNM classification and the GRCPSC $(4,5)$. Such a classification is probably based on Dukes' classification of colorectal cancer, which was published in the 1930s/1940s for colorectal cancer which was ranked as the leading cause of cancer-related death in Western countries. Dukes' classification is not only simple and clear but it also correlates extremely well with prognosis $(19,20)$. Dukes' system classifies colorectal cancer as Dukes' B or Dukes' C according to the presence or absence of lymph node metastases, indicating that the clinical importance of the $\mathrm{N}$ factor was recognized approximately 80 years ago. Dukes' method has long been used as a classification that reflects both tumor staging and progression and it has made a significant contribution to patient management $(19,20)$.

Most colorectal cancers are moderately or well-differentiated adenocarcinomas, and the main sites of recurrence/ metastasis are the liver and lungs. This is more correct for colon cancer, while local recurrence involving the pelvic floor is also common for rectal cancer (2). Recurrence by peritoneal dissemination or bone metastasis is relatively rare, and distant hematogenous metastases are closely associated with involvement of the portal vein, so that the presence or absence of lymph node metastasis is a good indicator of patient prognosis (2-5). In contrast, undifferentiated/poorly differentiated and highly malignant cancers such as esophageal cancer and gastric cancer show various forms of recurrence including peritoneal dissemination, mediastinal lymph node metastasis, and bone metastasis. For the management of breast cancer, surgical treatment, postoperative adjuvant therapy such as chemotherapy and/or radiation therapy, and treatment of recurrence are all subdivided, making the subcategorization of stage-specific treatment protocols quite complicated (4,6-8). Therefore, while there has been a significant improvement in the outcome of postoperative adjuvant therapy and treatment of recurrence for various types of cancer, in order to identify systemic disease in the early postoperative period, consideration of the $\mathrm{T}$ category is the second most important factor after the $\mathrm{N}$ category $(4,6-8)$. Since vascular/lymphatic invasion, which precedes lymph node metastasis, is due to tumor factors such as local expansion/infiltration, a classification based on the T-factor (mainly on $\geq \mathrm{T} 3+$ the $\mathrm{N}$ factor) should be practical and accurate for determining the disease stage and tumor progression (4).

In this study, analyses based on the $\mathrm{N}$ factor revealed no significant differences in the 5Y-RFS rate between the T1/2 and the $\geq \mathrm{T} 3$ category when comparing stage IIIa with IIIb. RFS is generally considered to be useful for assessing the treatment response during the first 6 months after surgery in patients receiving first-line postoperative adjuvant therapy. 3Y-RFS and 5Y-RFS are also suitable for the evaluation of recurrence/metastasis, but it has been reported that comparison of $5 \mathrm{Y}-\mathrm{OS}$ is more appropriate for assessing the outcome of each stage/subcategory. This is because $5 \mathrm{Y}-\mathrm{OS}$ assesses all outcomes, including the results of treatment after metastasis/ recurrence, while 5Y-RFS has an endpoint determined by the presence or absence of recurrence $(17,18)$. In this study, the $5 \mathrm{Y}-\mathrm{OS}$ of $\mathrm{T} 1 / 2$ and $\geq \mathrm{T} 3$ cases showed significant differences $(\mathrm{p}=0.003 / \mathrm{p}=0.008)$, suggesting that the $\mathrm{N}$ factor is closely associated with the overall survival of patients in the stage III subcategories. Our N1 ( $\leq 3$ nodes) patients received D2 or more extensive dissection ( $\mathrm{D}$-number $>\mathrm{N}$-number) according to the GRCPSC and Japanese rules for dissection of lymph nodes during radical surgery, which was macroscopically curative $(3,5,21)$. On the other hand, while the number of patients in the T1/2N2 category was very small (only 9 patients in 15 years), no highly malignant, undifferentiated/poorly differentiated cancers were found ( 2 patients had tumors classified as well-differentiated, 6 were classified as moderately differentiated, and 1 was classified as other). It is presumed that $\mathrm{N} 2$ lymph node metastases ( $\geq 4$ nodes) would eventually develop into systemic disease regardless of the $\mathrm{T}$ factor, i.e., even when the primary tumor is small and the depth of invasion is shallow. Future investigation should therefore elucidate the mechanism by which cancer cells spread from the primary tumor to nearby lymph nodes, eventually resulting in systemic metastasis. Our analysis based on the $\mathrm{T}$ factor, comparing $\mathrm{T} 1 / 2$ with $\geq \mathrm{T} 3$ in stage IIIa patients, revealed significant differences in both 5Y-RFS and 5Y-OS, but no significant differences were found in stage IIIb patients. This clearly suggests that the T1/2 category of disease up to stage IIIa is never more extensive than regional, involving the nearby mesenteric lymph nodes. When T1/2 patients were classified according to tumor size $(\leq 2 \mathrm{~cm} />2 \mathrm{~cm})$, it was found that $37.8 \%$ (17/45) of stage IIIa patients had tumors $\leq 2 \mathrm{~cm}$, while only $11.1 \%$ (1/9) of stage IIIb patients had tumors $\leq 2 \mathrm{~cm}$ (NS; data not shown). Furthermore, the percentage of patients with tumors $\leq 5 \mathrm{~cm}$ was $91.1 \%$ (41/45) in stage IIIa and $66.7 \%$ (6/9) in stage IIIb [p=0.046; HR, 0.298 (95\% CI, 0.096-0.928; data not shown)]. Thus, most of our $\geq \mathrm{T} 3$ patients had tumors $>5 \mathrm{~cm}$. These results suggest that stage IIIA/IIIa patients who are N1 ( $\leq 3$ nodes) and T1/2 patients with tumors mainly localized to the primary site and relatively small lesions $(\leq 5 \mathrm{~cm})$ would be better subcategorized as stage I, which would indicate a more favorable prognosis with a $5 \mathrm{Y}-\mathrm{OS}$ of $90 \%$ or better (4).

It is thought that lethal hematogenous metastasis to the liver or lungs occurring after curative resection of stage II/III cancer is due to residual cancer cells circulating through the body during the perioperative period. These cells escape from the immune system and reside in organs such as the liver or 
lungs where a nurturing microenvironment allows cancer cells to proliferate $(22,23)$. Various studies have found a close relationship between recurrence/metastasis and floating occult neoplastic cells (ONCs) detected by cytokeratin immunohistochemistry in the sinuses of lymph nodes distant from the primary tumor (24-28). ONCs can be counted after immunostaining, which identifies these malignant cells trapped in the lymph node sinuses in a part of the body's immune defense mechanism $(22,23)$. ONCs are classified as single cells (up to 3 floating ONCs), clusters (2-20 ONCs), and mixed single cells + clusters [both solitary tumor cells and micro-aggregates $(\leq 0.2 \mathrm{~mm})$ of 2-20 tumor cells] $(29,30)$. In particular, ONCs classified as single cells + clusters in patients with stage III colorectal cancer show a high sensitivity/high specificity/high negative predictive value for distant metastasis/recurrence. Therefore, ONCs may contribute to the screening of patients with a high risk of recurrence in the early postoperative period. This would also reduce the mental burden on patients in the low-risk group and aid in the planning of appropriate follow-up. In patients with stage IIIA/IIIa cancer categorized as T1/2N1 ( $\leq 3$ nodes), tumor cells may be almost completely eliminated by adjuvant chemotherapy and host antitumor immunity, although single cells or small clusters may continue to circulate in the body (14-17). It has been reported that a true survival benefit is achieved when there is improvement of survival after recurrence (21-23). Thus, it may be important during postoperative adjuvant chemotherapy to accurately detect occult systemic disease by identification of viable free cancer cells such as floating clusters and aggregates, and to eliminate these cells in the perioperative period before distant metastasis/ recurrence occurs $(29,30)$. It would be ideal to use targeted multi-drug combination regimens for adjuvant chemotherapy along with the standard 5-FU-based anticancer agents to attain sufficient efficacy (15-17). In the future, we must focus on the comparison of $\geq \mathrm{T} 3$ cases between stage IIIa and IIIb excluding those categorized as T1/2 with regard to the relationship between ONCs; single cells + clusters and recurrence/ metastasis. Finally, if floating clusters are important for predicting distant metastases/recurrence, then we must conduct detailed investigation of the sensitivity of such clusters to anticancer agents as well as the antitumor effect of moleculartargeting agents such as bevacizumab + FOLFOX for postoperative adjuvant chemotherapy of systemic disease such as N2 ( $\geq 4$ metastatic nodes) disease.

\section{Acknowledgements}

This study was supported by grants from the Occult Neoplastic Cell Research and Study Group (no. 2010-5007; Tokai University Hachioji Hospital, Hachioji, Tokyo, Japan) and the Research and Study Program of Tokai University Educational System General Research Organization (no. 2007-04; Tokai University Hospital, Isehara, Kanagawa, Japan).

\section{References}

1. Cases treated in 1994. In: Multi-Institutional Registry of Large Bowel Cancer in Japan. Vol. 23. Japanese Society for Cancer of the Colon and Rectum, Tokyo, 2002.

2. Makuuchi M and Sugihara K (eds): Knacks and Pitfalls: Surgery of the Colon, Rectum and Anus. 2nd edition. Bunkoudou Co. Ltd., Tokyo, 2004
3. Treatment Guideline for the Large Bowel Cancer in Japan. Japanese Society for Cancer of the Colon and Rectum, Tokyo, 2010.

4. TNM Classification of Malignant Tumours. 7th edition. John Wiley and Sons, Ltd., publication, New York, 2009.

5. General Rules for Clinical and Pathological Studies on Cancer of the Colon, Rectum and Anus. Japanese Society for Cancer of the Colon and Rectum. 7th edition and revised version 2009. Kanehara \& Co., Ltd., Tokyo, 2006.

6. National Institutes of Health Consensus Development Panel. National Institutes of Health Consensus Development Conference Statement: Adjuvant Therapy for Breast Cancer, November 1-3, 2000. J Natl Cancer Inst 93: 979-989, 2001.

7. Goldhirsch A, Glick JH, Gelber RD, Coates AS and Senn HJ: Meeting highlights: International Consensus Panel on the Treatment of Primary Breast Cancer. J Clin Oncol 19: 3817-3827, 2001.

8. Abrams JS: Adjuvant therapy for breast cancer - results from the USA consensus conference. Breast Cancer 8: 298-304, 2001.

9. Health and Welfare Statistics Association. J Health Welfare Stat 57: 48-51, 2010/2011.

10. Mukai M, Sato S, Tajima T, Kimura T, Komatsu N, Ninomiya H, Nakasaki H, Ogoshi K and Makuuchi H: Predicting recurrence and metastasis of Dukes' A primary colorectal cancer with or without proper muscle invasion. Oncol Rep 12: 1305-1308, 2004.

11. Mukai M, Tajima T,Nakasaki H, Sato S, Ogoshi K and Makuuchi H: Efficacy of postoperative adjuvant oral immunochemotherapy in patients with Dukes' B colorectal cancer. Ann Cancer Res Ther 11: 201-214, 2003.

12. Mukai M, Sato S, Ninomiya H, Wakui K, Komatsu N, Tsuchiya K, Nakasaki H and Makuuchi H: Recurrence and 5-FU sensitivity of stage II/Dukes' B colorectal cancer with occult neoplastic cells in lymph node sinuses. Oncol Rep 14: 1171-1176, 2005.

13. Ito I, Mukai M, Ninomiya $\mathrm{H}$, Kishima $\mathrm{K}$, Tsuchiya $\mathrm{K}$, Tajima $\mathrm{T}$, Oida Y, Nakamura M and Makuuchi H: Comparison between intravenous and oral postoperative adjuvant immunochemotherapy in patients with stage II colorectal cancer. Oncol Rep 20: 1189-1194, 2008.

14. Mukai M, Tajima T, Nakasaki H, Sato S, Ogoshi K and Makuuchi H: Efficacy of postoperative adjuvant oral immunochemotherapy in patients with Dukes' C colorectal cancer. Ann Cancer Res Ther 11: 215-229, 2003

15. Mukai M, Sato S, Ninomiya H, Wakui K, Tsuchiya K, Komatsu N, Nakasaki $\mathrm{H}$ and Makuuchi M: Recurrence and 5-FU sensitivity of stage III/Dukes' C colorectal cancer with occult neoplastic cells in lymph node sinuses. Oncol Rep 14: 1165-1169, 2005.

16. Mukai M, Sato S, Ninomiya H, Wakui K, Komatsu N, Matsui N, Nakamura M, Nakasaki H and Makuuchi H: Sensitivity to CPT-11 and platinum derivatives for stage III/Dukes' C colorectal cancer with occult neoplastic cells in lymph node sinuses. Oncol Rep 17: 1027-1032, 2007.

17. Mukai M, Ninomiya H, Kishima K, Tsuchiya K, Tajima T, Ito I, Nakamura $\mathrm{M}$ and Makuuchi M: Efficacy of 5FU/LV + CPT-11 as the 1st-line postoperative adjuvant chemotherapy in patients with stage IIIa colorectal cancer. Oncol Rep 22: 621-629, 2009.

18. Mukai M, Kishima K, Uchiumi F, Ishibashi E, Tajima T, Fukasawa M, Nakamura M and Hiroyasu Makuuchi: Comparison of QOL and adverse events for postoperative adjuvant chemotherapy in outpatients with node-positive colorectal or gastric cancer. Oncol Rep 21: 1061-1066, 2009.

19. Dukes $C$ (Subsection in paper by Gordon-Watson $C$ and Dukes C): The treatment of carcinoma of the rectum with radium: with an introduction on the spread of cancer of the rectum. Br J Surg 17: 643-648, 1930.

20. Dukes CE: Cancer of the rectum: an analysis of 1000 cases. J Pathol 50: 527-539, 1940.

21. Mukai M, Ito I, Mukoyama S, Tajima T, Saito Y, Nakasaki H, Sato $\mathrm{S}$ and Makuuchi H: Improvement of 10 -year survival by Japanese radical lymph node dissection in patients with Dukes' B and C colorectal cancer: a 17-year retrospective study. Oncol Rep 10: 927-934, 2003.

22. Mukai M, Sato S, Komatsu N, Nishida T, Shiba K, Ito I, Nakasaki H and Makuuchi H: Correlation between occult neoplastic cells in the lymph node sinuses and recurrence in patients with Dukes' C colorectal cancer. Oncol Rep 10: 1165-1169, 2003.

23. Mukai M, Sato S, Komatsu N, Nishida T, Shiba K, Ito I, Nakasaki $\mathrm{H}$ and Makuuchi H: Correlation between occult neoplastic cells in the lymph node sinuses and recurrence in patients with curatively resected Dukes' B colorectal cancer. Oncol Rep 10: 1177-1181, 2003. 
24. Mukai M, Sato S, Nakasaki H, Saito Y, Nishiumi N, Iwasaki M, Tokuda Y, Ogoshi K, Inoue H and Makuuchi H: Occult neoplastic cells in the lymph node sinuses and recurrence of primary breast, lung, esophageal and gastric cancer. Oncol Rep 11: 81-84, 2004.

25. Mukai M, Sato S, Komatsu N, Kimura T, Ninomiya $H$, Nakasaki H, Ogoshi K and Makuuchi M: Accuracy of criteria for predicting the recurrence and metastasis of stage I and II gastric cancer without lymph node metastasis. Oncol Rep 12: $59-62,2004$

26. Mukai M, Sato S, Komatsu N, Kimura T, Ninomiya H, Nakasaki H, Ogoshi K and Makuuchi H: Accuracy of criteria for predicting recurrence and metastasis in stage II and III gastric cancer with lymph node metastasis. Oncol Rep 12: 63-66, 2004.

27. Mukai M, Sato S, Komatsu N, Kimura T, Ninomiya $H$ Nakasaki H, Ogoshi K and Makuuchi H: Predicting the recurrence/ metastasis of stage II and III breast cancer with lymph node metastasis. Oncol Rep 12: 303-306, 2004.
28. Mukai M, Sato S, Komatsu N, Kimura T, Ninomiya H, Nakasaki H,Ogoshi K and Makuuchi H: Predicting the recurrence/ metastasis of stage I and II breast cancer without lymph node metastasis. Oncol Rep 12: 745-748, 2004.

29. Sekido Y, Mukai M, Kishima K, Izumi H, Fukumitsu H Hoshikawa T, Tajima T, Tobita K, Nakamura M, Nakamura N and Ogoshi K: Occult neoplastic cells in the lymph node sinuses and recurrence/metastasis of stage II/Dukes' B colorectal cancer. Oncol Rep 25: 69-73, 2011.

30. Sekido Y, Mukai M, Kishima K, Izumi H, Fukumitsu H, Hoshikawa T, Tajima T, Tobita K, Nakamura M, Nakamura N and Ogoshi K: Occult neoplastic cells in the lymph node sinuses and recurrence/metastasis of stage III/Dukes' C colorectal cancer. Oncol Rep 25: 915-919, 2011. 